\title{
A Pattern-Reconfigurable Slot Antenna for IoT Network Concentrators
}

\author{
Le Huy Trinh ${ }^{1}$, Trong Nhan Le ${ }^{2}$, Robert Staraj ${ }^{2}$, Fabien Ferrero ${ }^{2}$ and Leonardo Lizzi ${ }^{2, *}$ \\ 1 University of Information and Technology, National University Ho Chi Minh City, 700000 Ho Chi Minh City, \\ Vietnam; huytl@uit.edu.vn \\ 2 University Côte d'Azur, CNRS, LEAT, 06903 Sophia Antipolis, France; trong-nhan.le@unice.fr (T.N.L.); \\ robert.staraj@unice.fr (R.S.); fabien.ferrero@unice.fr (F.F.) \\ * Correspondence: leonardo.lizzi@unice.fr
}

Received: 27 September 2017; Accepted: 21 November 2017; Published: 29 November 2017

\begin{abstract}
In this paper, a directional pattern-reconfigurable slot antenna at $2.4 \mathrm{GHz}$ is presented. The antenna can provide three evenly-separated directional patterns and one omnidirectional pattern. The proposed solution is fully suitable for the integration in Internet of things (IoT) network base stations to increase the communication range and reduce packet collisions. The performance of the proposed antenna is numerically and experimentally assessed.
\end{abstract}

Keywords: reconfigurable antennas; directional antennas; Internet-of-things (IoT); PIN diodes

\section{Introduction}

The term Internet of things (IoT) has attracted the attention of researchers and technology companies throughout the world. IoT refers to a network that has the ability to sense the environment as well as to be controlled remotely through a wireless connection. In the last years, thanks to the evolution of communication technologies, IoT networks have undergone remarkable progress. As the mentioned in the Cisco report [1], the amount of connected devices will increase dramatically from 8.7 billion in 2012 up to 50 billion in 2020. This trend is leading to the creation of the so-called smart environments, such as smart homes, smart cities, and smart transportation, etc. [2,3].

In such environments, the use of pattern reconfigurable antennas can remarkably improve the quality of the wireless communication. As a matter of fact, thanks to the possibility of steering the energy radiated by the antenna towards specific directions, several advantages can be obtained. They include a reduction of interferences since specific targets for communication can be selected, the prevention of complex multi-hop transmissions by increasing the node communication ranges, and the increase of data throughput by exploiting spatial reuse [4].

In the literature, several pattern reconfigurable antennas have been proposed. The classical approach consists in changing the radiation direction through the tuning of the phases of the elements of an antenna array. Such a solution, however, implies high costs, large dimensions, and structures with high power consumption. A different solution, more suitable for IoT network applications, relies on the integration of active electronic components in the antenna geometry. A simple approach consists in the combination of multiple radiating elements connected to a radio-frequency (RF) switch. Normally, the radiating element is designed as a directional antenna. Due to the operation of the switch, the radiation pattern of the overall antenna system can be changed by selecting the proper antenna element [5].

Ha et al. [6] have introduced a beam-steering antenna for wearable applications that can cover three different directions by using two RF switches. However, the small gain and the impossibility of covering the entire 360-degree plane limits the effectiveness of the antenna. Trying to solve these problems, a different reconfigurable antenna has been presented in [7]. With a gain of $1 \mathrm{dBi}$ for the 
omnidirectional mode and $3.5 \mathrm{dBi}$ for eight directional modes, this antenna represents a good choice for $2.4 \mathrm{GHz}$ wireless local area networks. Nevertheless, the small operation frequency range (from 2.4 to $2.48 \mathrm{GHz}$ ) can be a real limitation. Beside, several additional antennas have been proposed in the literature for wireless sensors networks (WSNs) or Picocell applications using sectorial pattern reconfiguration [8-11]. However, they also present some limitations. The solution in [8] shows a small gain (about $1.9 \mathrm{~dB}$ at $2.4 \mathrm{GHz}$ ), while others have narrow bandwidths $[9,10]$ (about $60-80 \mathrm{MHz}$ for a return loss lower than $-10 \mathrm{~dB}$ ), or dimensions that are not compatible with IoT applications [11] $\left(10 \times 100 \times 100 \mathrm{~mm}^{3}\right)$.

In this paper, a reconfigurable directional antenna suitable for IoT network base stations is presented. It is based on the use of a metallic cylinder in which three fonts are excited by a monopole radiating element. Each slot can be electromagnetically opened or closed through 3 PIN diode switches. By controlling the state of each switch, the antenna can radiate towards 3 different directions or can exhibit an omnidirectional radiation pattern.

\section{Reconfigurable Antenna Design}

The geometry of the proposed reconfigurable antenna is shown in Figure 1. It is constituted by a metallic cylinder closed at the bottom and at the top. Its base is used as ground plane for a monopole, which therefore remains inside the cylinder. On the lateral surface of the cylinder, three equally-separated rectangular slots are etched. A switch is put in the middle of each slot, connecting the two horizontal slot edges. The monopole is modeled as a metallic wire of $1 \mathrm{~mm}$ in diameter, while the thickness of the metal plates is $0.5 \mathrm{~mm}$.

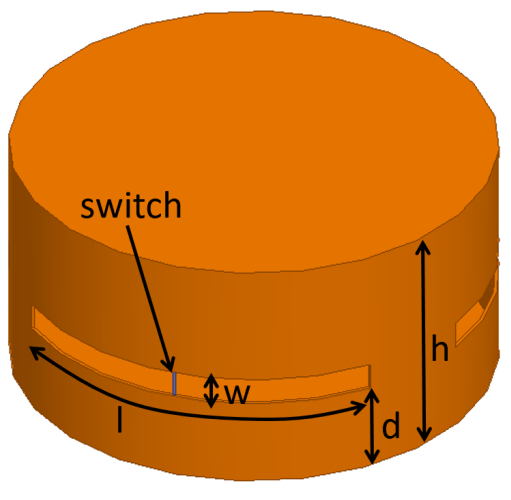

(a)

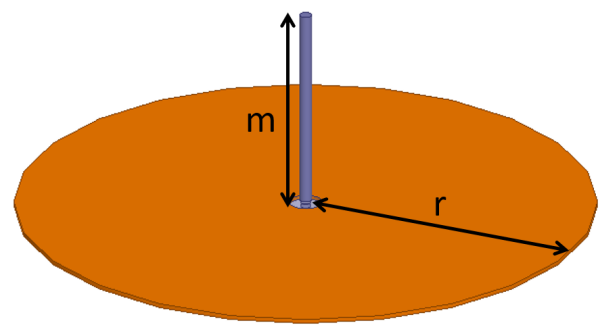

(b)

Figure 1. Proposed antenna geometry. (a) The external metallic cylinder with the slots, and (b) the internal monopole antenna used to excite the slots.

The antenna functioning is based on the fact that the monopole excites the slots, which radiate outside the cylinder. The slots, acting as magnetic dipoles, radiate a vertically polarized electromagnetic field. The radiation behavior of each slot depends on its dimensions. The length $l$ determines the slot resonant frequency, while the width $w$ defines the impedance matching. Consequently, the monopole length $m$ as well as the dimensions of the slots have been optimized through a trial-and-error procedure so that the antenna system can efficiently radiate at the operating frequency of $2.4 \mathrm{GHz}$.

The reconfiguration mechanism is based on the control of the switches. As a matter of fact, when the switch is open, it becomes electromagnetically transparent for the corresponding slot, which therefore radiates at the intended frequency. In contrast, when the switch is closed, the size of the slot is divided by two. This doubles the slot resonant frequency so that the $2.4 \mathrm{GHz}$ electromagnetic field is not radiated. 
The optimized values of the antenna geometrical parameters are summarized in Table 1 following the notation used in Figure 1. The overall antenna occupancy is given by a cylindrical volume of $70 \mathrm{~mm}$ diameter (corresponding to $0.57 \lambda$ at $2.45 \mathrm{GHz}$ ) and $38 \mathrm{~mm}$ in height $(0.31 \lambda$ at $2.45 \mathrm{GHz}$ ).

Table 1. Optimized values of the antenna geometrical parameters (in $\mathrm{mm}$ ).

\begin{tabular}{cccccc}
\hline $\boldsymbol{r}$ & $\boldsymbol{h}$ & $\boldsymbol{w}$ & $\boldsymbol{l}$ & $\boldsymbol{d}$ & $\boldsymbol{m}$ \\
\hline 35 & 38 & 4 & 50 & 15 & 24 \\
\hline
\end{tabular}

The antenna allows four different configurations depending on the states of the switches (Table 2). Figure 2 shows the simulated impedance matching of the optimized antenna. As can be observed, the Voltage Standing Wave Ratio (VSWR) is lower than 2 all over the $2.4 \mathrm{GHz}$ band in the $C_{000}$ configuration, while it slightly increases for $C_{011}$. As the antenna is symmetrical, the impedance matching for $C_{110}$ and $C_{101}$ is not reported. As for the radiation behavior, the corresponding simulated realized gain patterns are shown in Figure 3 in solid red curves. As can be seen, the antenna can exhibit three directive radiation patterns headed at $90^{\circ}, 210^{\circ}$ and $330^{\circ}$, and an omnidirectional one. The latter is obtained by putting all the switches in the open configuration. For the sake of completeness, the gain patterns in the vertical plane for the directions of the maxima obtained in the two different configurations $C_{000}$ and $C_{011}$ are shown in Figure 4.

Table 2. Switch configurations.

\begin{tabular}{ccccc}
\hline Conf. & Sw. 1 & Sw. 2 & Sw. 3 & Dir. \\
\hline$C_{011}$ & open & close & close & $\phi=90^{\circ}$ \\
$C_{101}$ & close & open & close & $\phi=210^{\circ}$ \\
$C_{110}$ & close & close & open & $\phi=330^{\circ}$ \\
$C_{000}$ & open & open & open & omni \\
\hline
\end{tabular}

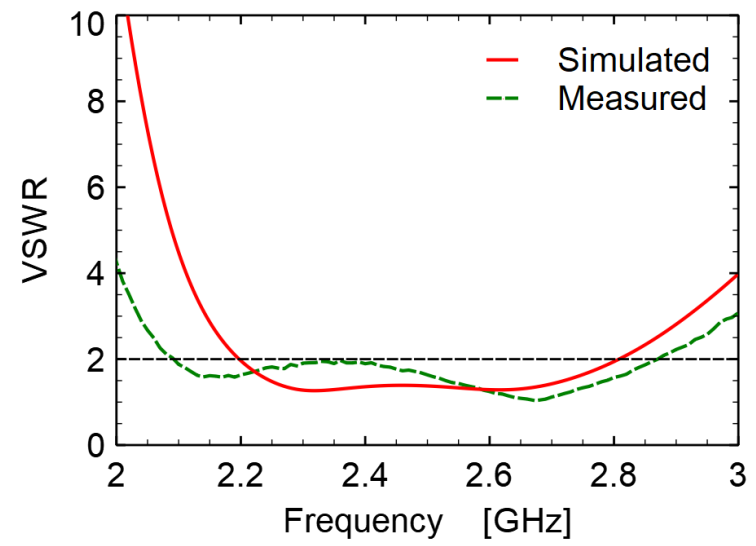

(a)

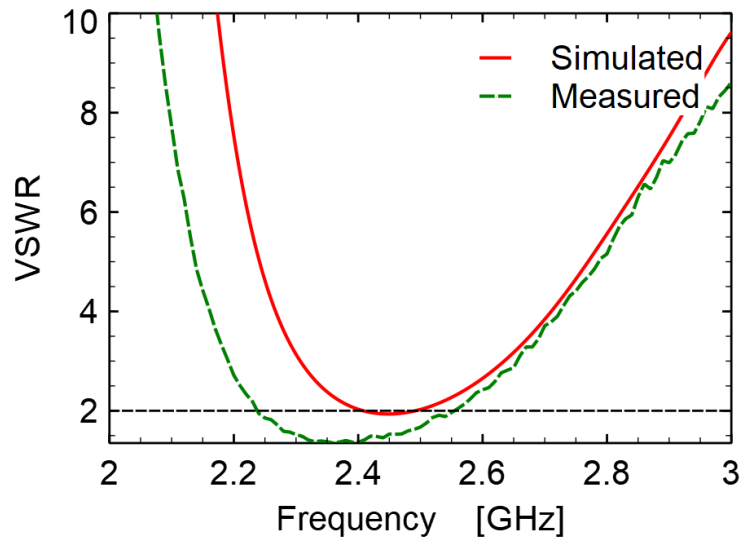

(b)

Figure 2. Simulated and measured impedance matching (VSWR values) of the proposed antenna in the (a) $C_{000}$ and (b) $C_{011}$ configurations. 


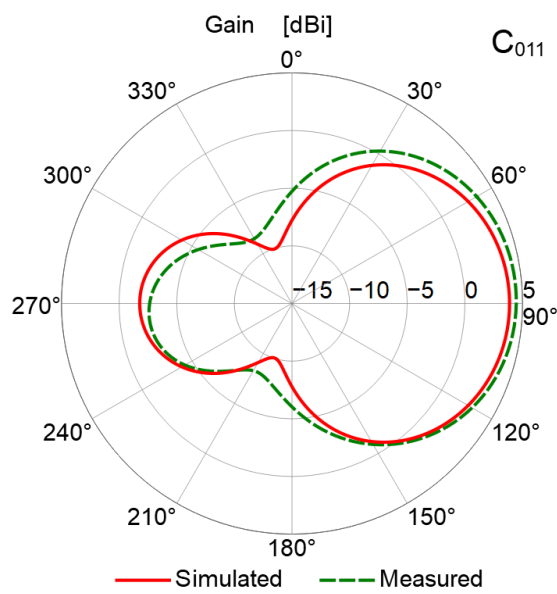

(a)

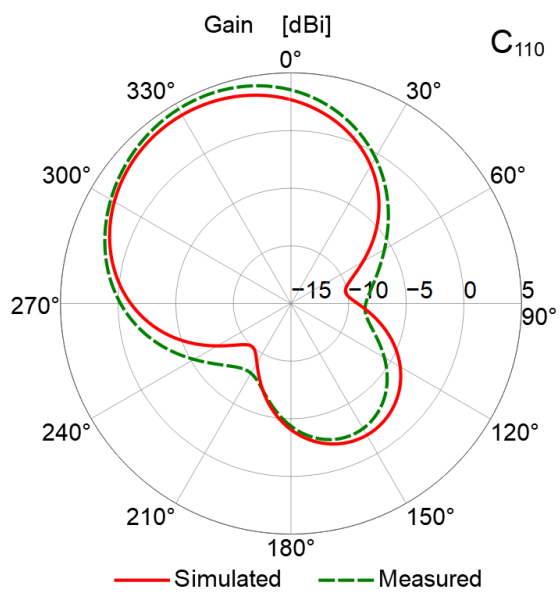

(c)

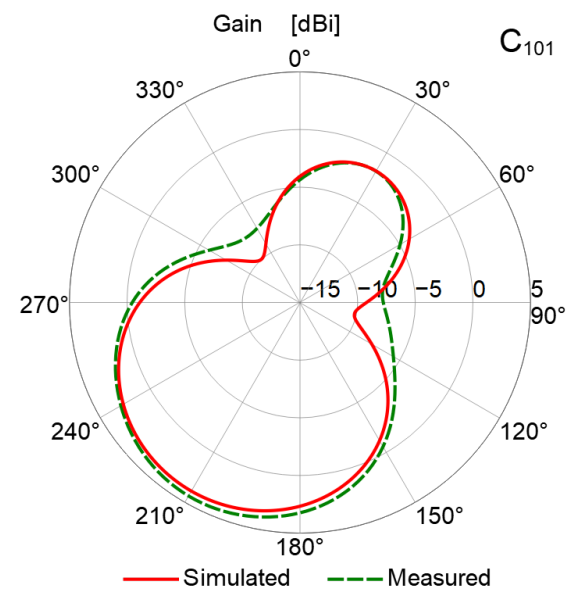

(b)

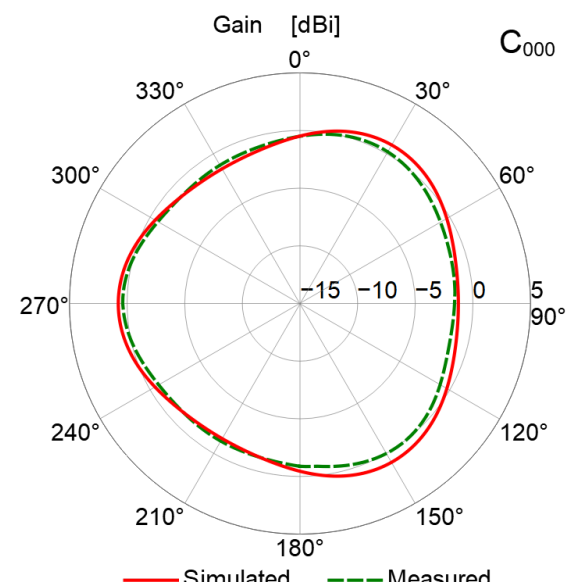

(d)

Figure 3. Simulated and measured realized gain patterns in the horizontal plane for the different switch configurations. (a) $C_{011}$, (b) $C_{101}$, (c) $C_{110}$, and (d) $C_{000}$.

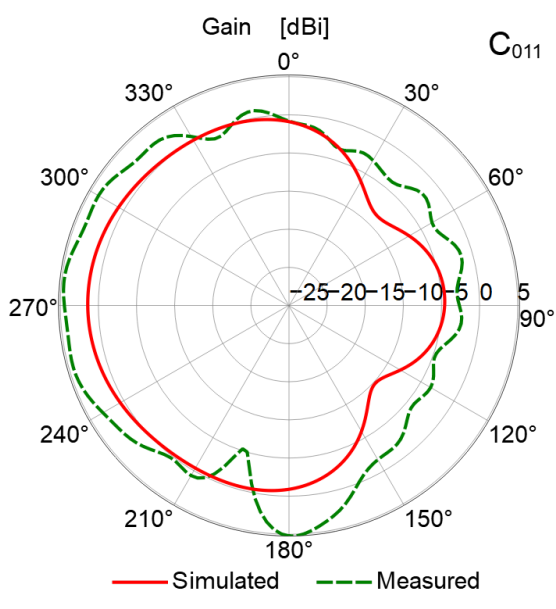

(a)

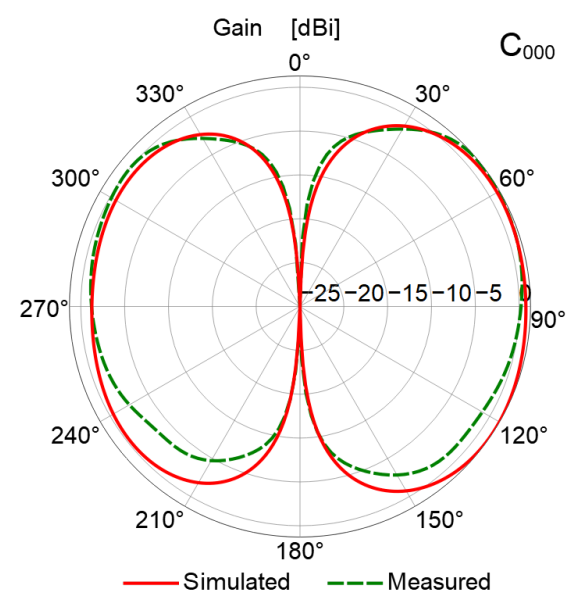

(b)

Figure 4. Simulated and measured realized gain patterns in the vertical plane for the configurations (a) $C_{011}$, and (b) $C_{000}$. 


\section{Experimental Validation}

\subsection{Antenna Prototype}

In order to experimentally validate the simulated electrical and radiating characteristics reported in the previous section, a prototype of the antenna has been created (Figure 5). The prototype is fabricated from a metallic cylinder in which the slots have been cut. A standard SubMiniature version A (SMA) connector has been soldered at center of the bottom face of the cylinder and was used to feed the internal monopole. The brown metal appearing at the bottom of the antenna is conductive duct tape, and it was used to close the antenna structure after the insertion of the monopole.

As for the switches, they have been created by using PIN diodes from MACOM (ref. MA4SPS502, pack. ODS-1270, MACOM Technology Solutions, Lowell, MA, USA) with a very small package in order to limit as much as possible the impact on the slot radiation. When the diode is reverse-biased, its resistance is very high $(>1 \mathrm{k} \Omega)$ and the diode behaves like an open circuit. Otherwise, its resistance becomes very low (around $1 \Omega$ ) as a short circuit. DC voltage to bias the PIN diode is provided from a control system, such as a microcontroller.

When the PIN diode is biased, to ensure that the DC source does not interfere with the RF source, the DC and RF blocks are integrated. A capacitor has been used to isolate the RF source from the current that activates the PIN diode as shown in Figure 6. The PIN diode is forward- biased with $10-\mathrm{mA}$ current, 3-V voltage, and a $250 \Omega$ resistor, which induces a power consumption of $30 \mathrm{~mW}$. Similarly, the RF block, which is a choke coil, attenuates the high frequencies completely.

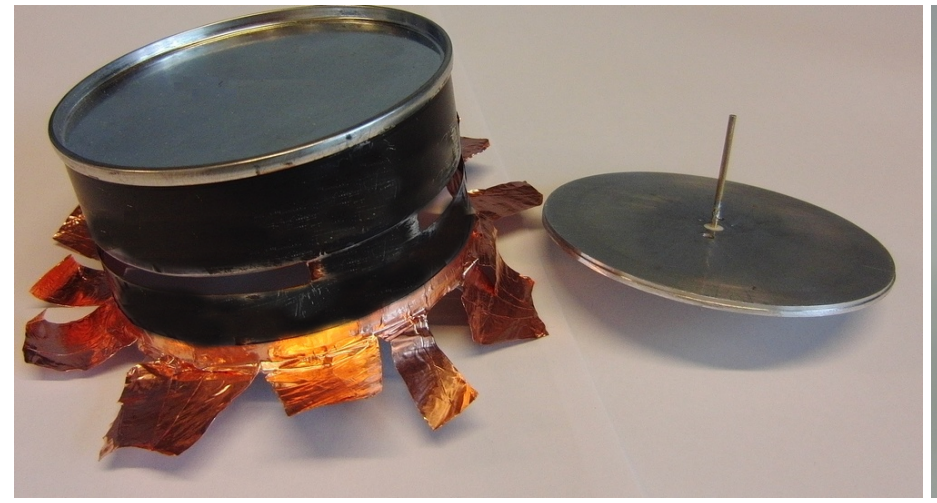

(a)

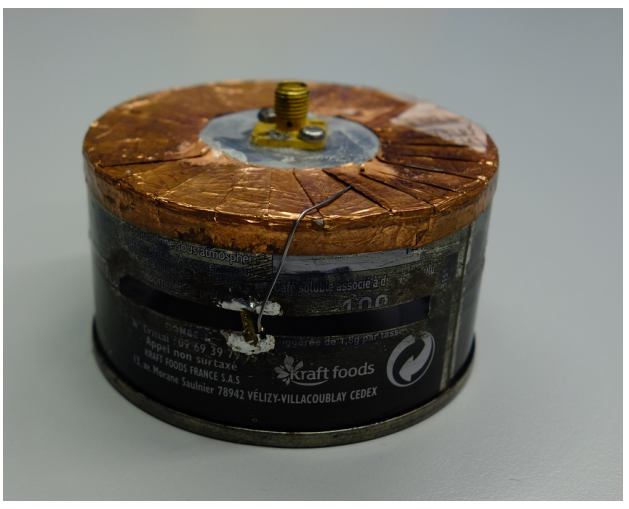

(b)

Figure 5. Pictures of the prototype. (a) top view (b) bottom view.

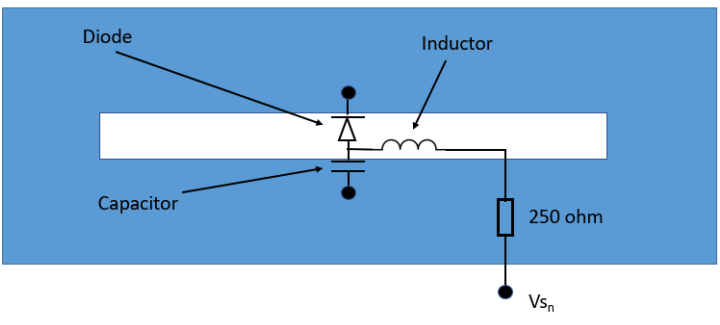

(a)

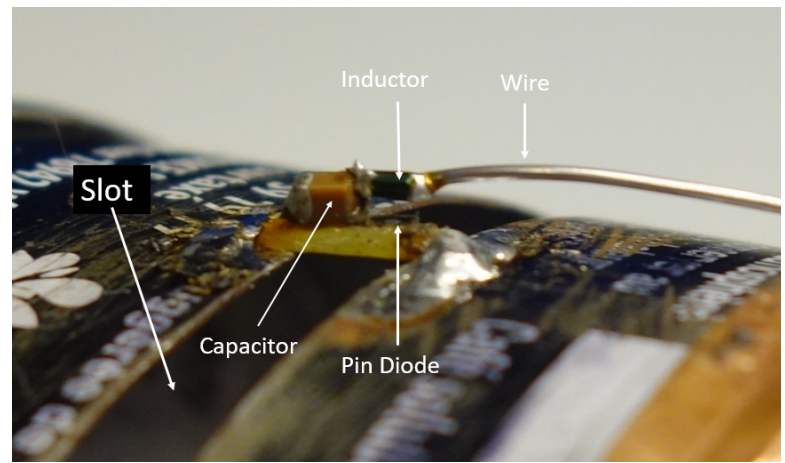

(b)

Figure 6. (a) Schematic and (b) zoom picture on the switching module placed in the slot. 


\subsection{Measurements}

Figure 2 shows the measured impedance matching of the realized prototype (green dashed curve). The VSWR has been measured in the $C_{000}$ and $C_{011}$ configurations, and in a non-controlled environment. Despite some differences with the simulated data given by some realization imperfections and by the non-ideal measurement environment, the prototype is well-matched at the operating frequency of $2.4 \mathrm{GHz}$. The measured operating bandwidth for which the $V S W R \leq 2 \mathrm{~dB}$ is between 2.1 and $2.9 \mathrm{GHz}$ for $C_{000}$ and between 2.25 and $2.55 \mathrm{GHz}$ for $C_{011}$. The experimental assessment of the prototype radiation characteristics has been performed using a Satimo Starlab station. The measured radiation patterns (total realized gain) in the horizontal and vertical planes for the different switch configurations are reported in Figures 3 and 4 (green dashed curves), respectively. As can be observed, the prototype is clearly capable of reconfiguring the radiation pattern towards three different directions. These correspond to the directions identified by the switches in the open configuration. For all the three directive configurations, the maximum gain is about $4 \mathrm{dBi}$ and the front-to-back ratio is almost $6 \mathrm{~dB}$. When all the diodes are in the open circuit mode, the antenna exhibits an omnidirectional behavior with a maximum variation smaller than $2 \mathrm{~dB}$. Finally, it is worth noting that, whatever the switch configuration, the measurements are in a very good agreement with the numerical data. The measured total efficiency of the antenna system is higher than $80 \%$ between 2.3 and $2.6 \mathrm{GHz}$.

To highlight the effectiveness of the proposed solutions, Table 3 reports a comparison with other existing state-of-the-art pattern-reconfigurable antennas. As can be observed, the proposed antenna is the smallest, while providing a larger operating bandwidth. Most important, it is the only one also providing an omnidirectional radiation pattern, which can be very useful in dealing with IoT network applications.

Table 3. Comparison with the state-of-the-art.

\begin{tabular}{cccccccc}
\hline Ref & $\begin{array}{c}\text { Freq } \\
(\mathbf{G H z})\end{array}$ & $\begin{array}{c}\text { Max Size } \\
(\lambda)\end{array}$ & N Sectors & $\begin{array}{c}\text { Min BW } \\
(\%)\end{array}$ & $\begin{array}{c}\text { Gain } \\
(\mathbf{d B i})\end{array}$ & $\begin{array}{c}\text { Eff } \\
\mathbf{( \% )}\end{array}$ & Omni \\
\hline$[12]$ & 2.4 & 0.83 & 8 & 4 & 5 & - & no \\
{$[13]$} & 2.4 & 0.5 & 4 & 3.5 & 6 & 84 & no \\
{$[14]$} & 0.55 & 0.7 & 4 & 10 & 5 & - & no \\
This work & 2.4 & 0.58 & 3 & 13 & 4 & 80 & yes \\
\hline
\end{tabular}

\section{Conclusions}

In this paper, a directional reconfigurable antenna suitable for IoT network base stations has been proposed. The antenna can exhibit three directional radiation patterns (a 4-dBi maximum gain and a 6-dB front-to-back ratio) and an omnidirectional pattern. The effectiveness of the proposed antenna has been experimentally demonstrated.

Future work will be aimed at increasing the number of possible directional patterns as well as reducing the antenna dimensions.

Acknowledgments: The authors would like to thank the CREMANT for its support in measurements.

Author Contributions: L.H. Trinh and L. Lizzi worked on the design of the antenna. T.N. Le and F. Ferrero worked on the control of the reconfiguration mechanism. R. Staraj supervised the entire work.

Conflicts of Interest: The authors declare no conflict of interest.

\section{References}

1. Evans, D. The internet of things. How the next evolution of the internet is changing everything. Cisco White Pap. 2011, 1, 1-11. 
2. Vlacheas, P.; Giaffreda, R.; Stavroulaki, V.; Kelaidonis, D.; Fonteinos, V.; Poulios, G.; Demestichas, P.; Somov, A.; Biswas, A.B.; Moessner, K. Enabling smart cities trhough a cognitive management framework for the internet of things. IEEE Commun. Mag. 2013, 51, 102-111.

3. Jin, J.; Gubbi, J.; Marusic, S.; Palaniswami, M. An information framework for creating a smart city through internet of things. IEEE Int. Things J. 2014, 1, 112-121.

4. Skiani, G.E.D.; Mitilineos, S.A.; Thomopoulos, S.C.A. A study of the performance of wireless sensor networks operating with smart antennas. IEEE Antennas Propag. Mag. 2012, 54, 50-67.

5. Catarinucci, L.; Guglielmi, S.; Colella, R.; Tarricone, L. Pattern-Reconfigurable Antennas and Smart Wake-Up Circuits to Decrease Power Consumption in WSN Nodes. IEEE Sens. J. 2014, 14, 4323-4324.

6. Ha, S.-J.; Jung, Y.-B.; Kim, Y.; Jung, C.W. Reconfigurable Beam-Steering Antenna Using Dipole and Loop Combined Structure for Wearable Applications. ETRI J. 2012, 34, 1-8.

7. Facco, M.; Piazza, D. Reconfigurable Zero-Order Loop Antenna. In Proceedings of the IEEE Antennas and Propagation Society International Symposium, Chicago, IL, USA, 8-14 July 2012; pp. 1-2.

8. Ramadan, A.; Al-Husseini, M.; Tawk, Y.; Kabalan, K.Y.; El-Hajj, A. A Novel Frequency/Pattern-Reconfigurable Microstrip Antenna for WLAN Applications. In Proceedings of the European Conference on Antennas and Propagation (EuCAP), Barcelona, Spain, 12-16 April 2010; pp. 1-3.

9. Ha, S.J.; Jung, C.W.; Kim, Y. Reconfigurable 3D Beam Steering for Intelligent Antenna System. Microw. Opt. Technol. Lett. 2011, 53, 2615-2619.

10. Hwang, K.S.; Ahn, J.; Kim, K.-J.; Yoon, H.K.; Yoon, Y.J. Pattern reconfigurable antenna for a wireless sensor network sink node. In Proceedings of the Asia Pacific Microwave Conference (APMC), Yokohama, Japan, 7-10 December 2010; pp. 2021-2024.

11. Kim, K.; Hwang, K.; Ahn, J.; Yoon, Y. Pattern Reconfigurable Antenna for Wireless Sensor Network System. Electron. Lett. 2012, 48, 984-985.

12. Catarinucci, L.; Guglielmi, S.; Colella, R.; Tarricone, L. Compact Switched-Beam Antennas Enabling Novel Power-Efficient Wireless Sensor Networks. IEEE Sens. J. 2014, 14, 3252-3259.

13. Le, T.N.; Pegatoquet, A.; Le Huy, T.; Lizzi, L.; Ferrero, F. Improving Energy Efficiency of Mobile WSN Using Reconfigurable Directional Antennas. IEEE Commun. Lett. 2016, 20, 1243-1246.

14. Kittiyanpunya, C.; Krairiksh, M. A Four-Beam Pattern Reconfigurable Yagi-Uda Antenna. IEEE Trans. Antennas Propag. 2013, 61, 6210-6214.

(C) 2017 by the authors. Licensee MDPI, Basel, Switzerland. This article is an open access article distributed under the terms and conditions of the Creative Commons Attribution (CC BY) license (http://creativecommons.org/licenses/by/4.0/). 